\title{
A Model Based on the Combination of IFN- $\gamma$, IP-10, Ferritin and 25-Hydroxyvitamin $D$ for Discriminating Latent From Active Tuberculosis in Children
}

OPEN ACCESS

Edited by:

Xiao-Yong Fan,

Fudan University, China

Reviewed by:

John Zaunders,

St Vincent's Hospital Sydney, Australia Hazel Marguerite Dockrell,

London School of Hygiene \& Tropical Medicine (LSHTM), United Kingdom Nadia Caccamo,

University of Palermo, Italy

${ }^{*}$ Correspondence:

Tomas M. Perez-Porcuna tomas.perez.porcuna@gmail.com

${ }^{+}$Co-senior authors

Specialty section:

This article was submitted to Infectious Diseases,

a section of the journal

Frontiers in Microbiology

Received: 13 December 2018

Accepted: 29 July 2019

Published: 14 August 2019

Citation:

Comella-del-Barrio P, Abellana R, Villar-Hernández $R$, Jean Coute $M D$, Sallés Mingels B, Canales Aliaga L, Narcisse M, Gautier J, Ascaso C,

Latorre I, Dominguez J and Perez-Porcuna TM (2019) A Model Based on the Combination of IFN- $\gamma$, IP-10, Ferritin and 25-Hydroxyvitamin $D$ for Discriminating Latent From Active Tuberculosis in Children.

Front. Microbiol. 10:1855 doi: 10.3389/fmicb.2019.01855

\author{
Patricia Comella-del-Barrio ${ }^{1}$, Rosa Abellana ${ }^{2 t}$, Raquel Villar-Hernández', \\ Mariette Doresca Jean Coute ${ }^{3}$, Beatriz Sallés Mingels ${ }^{4}$, Lydia Canales Aliaga ${ }^{5}$, \\ Margareth Narcisse ${ }^{3}$, Jacqueline Gautier ${ }^{3}$, Carlos Ascaso², Irene Latorre', \\ Jose Dominguez ${ }^{1 \dagger}$ and Tomas M. Perez-Porcuna ${ }^{6 *+}$
}

'Research Institute Germans Trias i Pujol, CIBER Respiratory Diseases, Universitat Autònoma de Barcelona, Badalona, Spain, ${ }^{2}$ Department of Basic Clinical Practice, Faculty of Medicine, University of Barcelona, Barcelona, Spain, ${ }^{3}$ Saint-Damien Pediatric Hospital, Tabarre, Haiti, ${ }^{4}$ Radiology and Imaging Diagnose Department, Manso Primary Care Center, Barcelona, Spain, ${ }^{5}$ Radiology Service, Research Unit of the Mútua Terrassa Foundation, University Hospital Mútua Terrassa, Terrassa, Spain, ${ }^{6}$ Department of Pediatrics, Tuberculosis and International Health Care Unit, Primary Care and Mútua Terrassa University Hospital, University of Barcelona, Terrassa, Spain

In recent years, pediatric research on tuberculosis (TB) has focused on addressing new biomarkers with the potential to be used as immunological non-sputum-based methods for the diagnosis of TB in children. The aim of this study was to characterize a set of cytokines and a series of individual factors (ferritin, 25-hydroxyvitamin D [25(OH)D], parasite infections, and nutritional status) to assess different patterns for discriminating between active TB and latent TB infection (LTBI) in children. The levels of 13 cytokines in QuantiFERON-TB Gold In-Tube (QFT-GIT) supernatants were analyzed in 166 children: 74 with active TB, 37 with LTBI, and 55 uninfected controls. All cytokines were quantified using Luminex or ELISA. Ferritin and 25(OH)D were also evaluated using CLIA, and Toxocara canis Ig-G antibodies were detected with a commercial ELISA kit. The combination of IP-10, IFN- $\gamma$, ferritin, and 25(OH)D achieved the best diagnostic performance to discriminate between active TB and LTBI cases in children in relation to the area under receiver operating characteristic $(R O C)$ curve 0.955 (confidence interval 95\%: 0.91-1.00), achieving optimal sensitivity and specificity for the development of a new test (93.2 and 90.0\%, respectively). Children with TB showed higher ferritin levels and an inverse correlation between $25(\mathrm{OH}) \mathrm{D}$ and IFN- $\gamma$ levels. The model proposed includes a combination of biomarkers for discriminating between active TB and LTBI in children to improve the accuracy of TB diagnosis in children. This combination of biomarkers might have potential for identifying the onset of primary TB in children.

Keywords: pediatrics, biomarkers, immune response, vitamin D, ferritin, enzyme-linked immunoassays, Mycobacterium tuberculosis, cytokines 


\section{INTRODUCTION}

At least one-quarter of the world's population is infected with Mycobacterium tuberculosis (World Health Organization [WHO], 2018a). Childhood tuberculosis (TB) represents at least $10 \%$ of the burden of the disease worldwide, being one of the most significant causes of childhood morbidity and mortality (World Health Organization [WHO], 2018b). Estimations show that there are far more children with TB globally than previously thought, with the majority being undiagnosed and untreated (Dodd et al., 2017). Underdiagnosis in children can occur for multiple reasons, such as the lack of a non-specific clinical presentation that delays seeking healthcare and diagnostic suspicion; the lack of a more accurate diagnostic test; and poor geographical and financial access to healthcare in some areas (Kyu et al., 2018; World Health Organization [WHO], 2018a).

Infants and young children have a higher risk of progressing to TB following a primary $M$. tuberculosis infection, usually due to a child being exposed to an infectious adult with active TB (Augustynowicz-Kopeć et al., 2012) and to the development of severe forms of the disease (Marais et al., 2004). Therefore, household detection of index TB cases, together with early detection of the infection and TB disease, followed by a prompt treatment, are fundamental in preventing disease progression (Dodd et al., 2018).

The diagnosis of pulmonary $\mathrm{TB}$ is a significant challenge in children due to the paucibacillary nature of the disease and the difficulties in expectorating. Although rapid molecular diagnosis represents a significant advance as an alternative to conventional microscopy and culture methods, its sensitivity remains unacceptably low in children since the technique is based on detecting M. tuberculosis from respiratory specimens (World Health Organization [WHO], 2018b). As for immunodiagnostic assays, both the tuberculin-skin-test (TST) and interferongamma release assays (IGRAs) - that measure cell-mediated immune responses following $M$. tuberculosis infection-are unable to distinguish between active TB and LTBI (Latorre and Domínguez, 2015) and limit the accuracy of M. tuberculosis detection (Dominguez et al., 2009; Velasco-Arnaiz et al., 2018). As a result, most active TB cases are diagnosed through clinical-and radiological when possible-scoring systems which have limitations due to the clinical presentation of the disease (Graham et al., 2015).

The immature immune system of young children added to the dynamic process of the host-pathogen interaction from M. tuberculosis infection, hamper defining the transition from latent infection to acute TB disease in children. Recently, a diversity of genetic and individual factors that may contribute to the outcome of the host immune set-points during M. tuberculosis infection have been described (Bastos et al., 2018). A good understanding of the diversity of these factors might be crucial to assess the impact of the host-pathogen interactions that occur during $M$. tuberculosis infection. In this sense, the WHO guidelines for the management of malnourished children, as well as guidelines for national TB programs, underline the importance of the association of malnutrition and TB in children (World Health Organization [WHO], 2013).
In addition, the impact of alterations in iron homeostasis and vitamin $\mathrm{D}$ deficiencies highlight profound effects on immune function and host defenses due to mechanisms that have not yet been resolved. Lastly, comorbidities by helminth infections impair the inflammatory and immune mechanisms involved in the control of M. tuberculosis infection (Ibrahim et al., 2017).

In recent years, pediatric research has focused on finding new biomarkers in non-sputum-based samples for the detection of M. tuberculosis infection (Nicol et al., 2015). Current studies have highlighted the importance of diverse forms of biomarkers with the potential to be used in immunological methods for the diagnosis of childhood TB (Togun et al., 2018). Furthermore, there is increasing data showing that modified interferon gamma release assays (IGRAs) based on the analysis of a combination of different markers could enhance the diagnostic accuracy (Chegou et al., 2014). However, only a few studies with small cohorts have identified some cytokine responses in QuantiFERON-TB Gold In-Tube (QFT-GIT, QIAGEN, Germany) supernatants which have the potential to monitor specific immunity against M. tuberculosis as candidate combinations of markers for the discrimination between LTBI and active TB in children (Togun et al., 2018). Despite these initial approaches, a clear pattern has not yet emerged, and the diagnostic performance of the cytokine biomarkers reported do not meet the minimum targets recommended by the WHO for a new diagnostic or triage test for TB in children (World Health Organization, 2017).

To our knowledge, no cytokine biomarkers based on QFT-GIT supernatants combined with individual factors can distinguish between active TB and LTBI in children. To improve the accuracy of the diagnosis of TB, the aim of this study was to characterize a set of cytokines and a series of individual factors (ferritin, 25-hydroxyvitamin D [25(OH)D], parasite infections, and nutritional status) and to assess different patterns for distinguishing between active TB and LTBI in children.

\section{MATERIALS AND METHODS}

\section{Study Population}

A prospective case-control study was conducted from August 2015 to December 2016 in the pediatric hospital of Saint Damien in Port-au-Prince (Haiti). Children (0-14 years old) who presented signs and symptoms compatible with active TB and/or documented TB exposure were screened for suspected TB. According to the hospital program, siblings (0-14 years old) of the children diagnosed with $\mathrm{TB}$ were also screened for TB or LTBI. Children (0-14 years old) from a school and a kindergarten were screened as uninfected controls. The exclusion criteria were: children with known immunodeficiency, on current immunosuppressive treatment, with a condition that could potentially compromise the immune system (e.g., children from oncology, rheumatology, nephrology and those who had undergone organ transplantation), children who had been under anti-TB treatment or preventive treatment during the previous year, and children not providing informed consent.

The following information was collected: age, sex, weight, height, previous medical history (including TB history, 
TB exposure, HIV status, and comorbidities, hemogram), vaccines, and current and previous medication (antibiotics, corticosteroids, antiparasitic drugs).

The following signs and symptoms were evaluated: cough and/or fever $\geq 2$ weeks (with no improvement after at least a 7-day course of amoxicillin), recent unexplained weight loss, and asthenia/fatigue. The TST was performed by a trained laboratory technician. Intradermal injection of $0.1 \mathrm{ml}$ of Tubersol (bioequivalent to 5 tuberculin units; Sanofi Pasteur, Toronto, ON, Canada) was placed into the ventral surface of the lower arm and read after $72 \mathrm{~h}$. A positive TST result was defined as an induration $\geq 10 \mathrm{~mm}$ in BCG-vaccinated children (those with a BCG scar), and $\geq 5 \mathrm{~mm}$ in non-BCG-vaccinated children or with a known adult TB contact (Bass et al., 1990; American Academy of Pediatrics Tuberculosis, 2009). A standardized specific $Z$ - score for detection of nutritional status weight-for-age (WAZ) was determined by WHO Anthro Plus 1.0.4 software (World Health Organization [WHO], 2009). Children with WAZ scores below -2 standard deviation (SD) were defined as underweight, and a WAZ score above 2 SD was defined as overweight.

A chest radiograph (anterior-posterior image) was performed in all the children screened for TB or LTBI. Chest radiographs were read by two external experts who were blinded to clinical data using a standardized reporting form and a third reader to resolve discordant opinions (Graham et al., 2015). In addition, nasopharyngeal aspirates and induced sputum were collected on three consecutive days for smear examination (auramine stain) by direct fluorescence microscopy in children suspected of having active TB. Histological examination was performed in children with suspicion of extrathoracic TB (ETB) with lymph node adenopathies.

Of all the children screened for TB or LTBI, only those with a positive TST result and/or microscopic confirmation were invited to participate in the study. Of all the children screened in the schools, only those with a negative TST result were invited to participate in the study as uninfected controls.

Treatment and preventive treatment were prescribed in all the children diagnosed with TB or LTBI, respectively. During $\mathrm{TB}$ treatment and preventive treatment, the patients were followed monthly until the end of treatment. Children with a positive sputum smear were retested at the fifth and sixth month of treatment.

\section{Definitions for Classification of the Children Enrolled in the Study}

Children were classified according to their clinical history, chest radiographs, smear examination, molecular diagnostic test, TST and QFT-GIT results. Active TB cases were defined as confirmed TB —children with relevant signs and symptoms and microbiologic confirmation of $M$. tuberculosis-, and unconfirmed TB - children without bacteriological confirmation but with relevant signs and symptoms, positive TST and/or QFT-GIT, radiological findings suggestive of TB, known TB contact, and clinical response to anti-TB treatment. Depending on the TB location, active TB cases were classified as ETB or intrathoracic TB. Children with intrathoracic TB were differentiated into those with pulmonary involvement (PTB) and children with isolated mediastinal lymphadenopathy in the absence of lung parenchyma involvement (Mediastinal TB). LTBI cases were defined as children with documented TB exposure, positive TST and/or QFT-GIT, normal chest radiographs, and no clinical signs of TB development in the last 6 months after diagnosis. Finally, uninfected controls were defined as asymptomatic children with no history of TB exposure, and negative TST and QFT-GIT.

\section{Laboratory Tests Performed Molecular Diagnostic Test}

Sputum samples were collected from children with microscopy confirmation and/or radiological findings to perform GeneXpert MTB/RIF (Cepheid, United States) according to the manufacturer's instructions.

\section{QuantiFERON-TB Gold In-Tube}

In all the participants, a $3 \mathrm{ml}$ blood sample was drawn for conventional QFT-GIT; the antigen tube (ESAT-6, CFP-10, and TB-7.7), and positive (phytohemagglutinin, mitogen) and negative (no antigen, nil) controls. Tubes were incubated at $37^{\circ} \mathrm{C}$ for $16-24 \mathrm{~h}$ and centrifuged according to the manufacturer's instructions. Plasma was harvested and stored at $-20^{\circ} \mathrm{C}$ until performing the enzyme-linked immunosorbent assay (ELISA). After the screening and recruitment of cases, plasma samples were sent to the laboratories of the Research Institute Germans Trias i Pujol (IGTP, Badalona, Spain) following optimum conservation conditions. Once there, the supernatants were analyzed and the results were interpreted according to the manufacturer's instructions.

\section{Ferritin, 25(OH)D, and Toxocara spp. Detection}

One milliliter of blood from all the participants was added to the biochemical tube to perform ferritin, 25(OH)D, and Toxocara canis determination. Ferritin and $25(\mathrm{OH}) \mathrm{D}$ concentrations were analyzed at the biochemistry laboratory of the Germans Trias i Pujol Hospital (Badalona, Spain) with a Chemiluminescence Immunoassay (CLIA) method using a Liaison instrument (DiaSorin Liaison, Stillwater, MN, United States). 25(OH)D is an indirect method to measure vitamin $\mathrm{D}$ in the blood. According to the literature, serum 25(OH)D levels equal or above $20 \mathrm{ng} / \mathrm{ml}$ were considered normal levels of vitamin D (Michael and Holick, 2007). To avoid variations during blood sampling, the blood was almost always collected on the same day of the week at approximately the same hour (13-15 h). Seasonal fluctuations did not affect sampling because the weather seasons in Haiti are barely defined. T. canis Ig-G antibodies were detected at the IGTP laboratory using a commercial ELISA kit (Ridascreen, R-Biopharm AG, Germany). Results with a sample index above 1.1 were considered positive according to the manufacturer's instructions.

\section{Detection of Soil-Transmitted Parasite Infections}

Stool samples were collected to detect intestinal parasites in all the participants. Stool samples were processed on the same day of collection at the laboratory of the pediatric 
hospital of Saint Damien using the Kato-Katz and formalingasoline technique (a modification of the formalin-ether sedimentation technique) as described previously (Katz et al., 1972; Ahmadi and Damraj, 2009) and were examined with optical microscopy.

\section{Cytokine Measurement}

Frozen supernatants remaining from the QFT-GIT tubes were used for the measurement of cytokine concentrations using a bead-based multiplex assay (Luminex 11-plex cytokine kit, R\&D Systems, United Kingdom) and measured by Bioplex manager software (version 5.0, Bio-Rad, United States) according to the manufacturer's instructions. After optimization experiments, granulocyte-macrophage colony-stimulating factor (GM-CSF), interferon (IFN)-gamma $(\gamma)$, interleukin (IL)-2, IL-5, IL-10, IL-13, IL-22, IL-17, and tumor necrosis factor (TNF)-alpha $(\alpha)$ were analyzed in a 1:8-fold dilution, while IL-1RA and induced protein (IP)-10 were analyzed in 1:8, 1:80, and 1:160-fold dilutions. In this way, values were within the detection limits marked in the standard curve. IL-32 and vascular endothelial growth factor (VEGF) were measured by DuoSet ELISA (R\&D Systems, United Kingdom) because of incompatibilities with the human custom multiplex cytokine kit. ELISA was performed according to the manufacturer's instructions.

\section{Statistical Analysis}

Qualitative variables were described using frequencies and percentages. Qualitative variables were described using median and interquartile ranges (IQR), or, using mean and standard deviation (SD) in case of variables were with a normally distribution, using mean and standard deviation. The independent variables analyzed were: sex, age, weight by age, BCG, hemoglobin levels, 25(OH)D levels, ferritin levels, the presence of intestinal helminths, IgG T. canis antibodies, and cytokines responses. The concentration of released cytokines released in response to $M$. tuberculosis antigens (Ag-TB) and phytohemagglutinin (PHA) was calculated by subtracting the concentration measured in the nil tube (Ag-TB, antigen minus nil; and PHA, mitogen minus nil). For all the variables with a normal distribution, the comparison between several groups was performed using the analysis of variance or Student's t-student test (only two groups). However, for variables non-normally distributed variables, the comparison between groups was performed using Kruskal-Wallis and Mann-Whitney tests. In the case of qualitative variables, comparisons were performed using the Fisher's exact test or chi-squared test. The Tukey method (normal distribution) or Benjamini and Hochberg method (non-normal distribution) was used to correct $p$-values in multiple comparisons. The cytokines that showed significant differences between study groups were evaluated and considered.

Multivariate logistic regression was performed to detect variables able to classify individuals among the three different study groups (active TB, LTBI, and uninfected). The forward method was used for selecting the best combinations of cytokines. Participants with a positive QFT-GIT result (TB and LTBI) were analyzed separately (Binomial) from those with a negative QFTGIT result (TB, LTBI, and uninfected; Multinomial). The results were expressed using the odds ratios (OR) and their confidence intervals. Sensitivity and specificity were calculated for assessing the value of performing a diagnostic test. The receiving operating characteristic (ROC) curve was used to evaluate diagnostic accuracy. The optimal cut-off value was identified to maximize the difference between true positive and false positives subjects. The level of significance was set at 0.05 .

These analyses were performed using the statistical software IBM SPSS Statistics v. 25 (SPSS, Chicago, IL, United States), R package v.3.0.5 (R Foundation for Statistical Computing, Vienna, Austria), and GraphPad PRISM v. 5 (GraphPad Software, Inc., San Diego, CA, United States).

\section{RESULTS}

\section{Study Subjects}

A total of 305 children suspected of having LTBI or TB were screened in the hospital, whereas a total of 96 uninfected children were screened in a school and a kindergarten. Among the patients screened at the hospital, 156 were recruited, and 149 were excluded because they did not meet the inclusion criteria (informed consent not obtained, negative TST). Of the 156 participants, $111(71.2 \%)$ were analyzed in the study, and 45 $(28,8 \%)$ were lost to follow-up and were therefore not included in the analysis due to lack of complementary tests necessary for clinical assessment (chest radiographs, biopsies). Among the children screened at school and kindergarten, 55 were recruited and analyzed, and 41 were excluded because they did not meet the inclusion criteria (informed consent not obtained, positive TST). Among the 166 children analyzed (111 screened at the hospital and 55 screened at school and kindergarten), 74 had active TB (44.6\%), 37 had LTBI (22.3\%), and 55 were uninfected (33.1\%). Of the 74 active TB cases, 8 had ETB, and 66 had intrathoracic TB. Among the intrathoracic TB children, 48 had PTB, and 18 had Mediastinal TB (Figure 1).

Table 1 shows the description, demographic, and clinical characteristics among study groups according to their QFT-GIT result. Significant differences were observed in age and body WAZ $z$-scores ( $p=0.001$ and $p=0.001$, respectively) among the study groups with different QFT-GIT results (positive, negative, and indeterminate). Whereas LTBI cases with positive QFT-GIT were older than active TB cases with negative QFT-GIT $(p=0.002)$, active TB cases with positive QFT-GIT had lower WAZ $z$-scores than uninfected controls $(p=0.001)$. However, comparisons among QFT-GIT results within each study group (TB, LTBI, and non-infected) were performed, and no significant differences were observed.

\section{Characterization of Subjects With a Positive QFT-GIT Test}

Comparisons between the groups with active $\mathrm{TB}$ and positive QFT-GIT were performed (Table 2). The Ag-TB levels of IL-17A, GM-CSF, together with the PHA levels of TNF- $\alpha$, GM-CSF and, IL-13, and median hemoglobin levels showed significant differences between active TB groups $(p=0.009, p=0.027$, $p=0.027, p=0.040$ and $p=0.042, p=0.006$, respectively). 


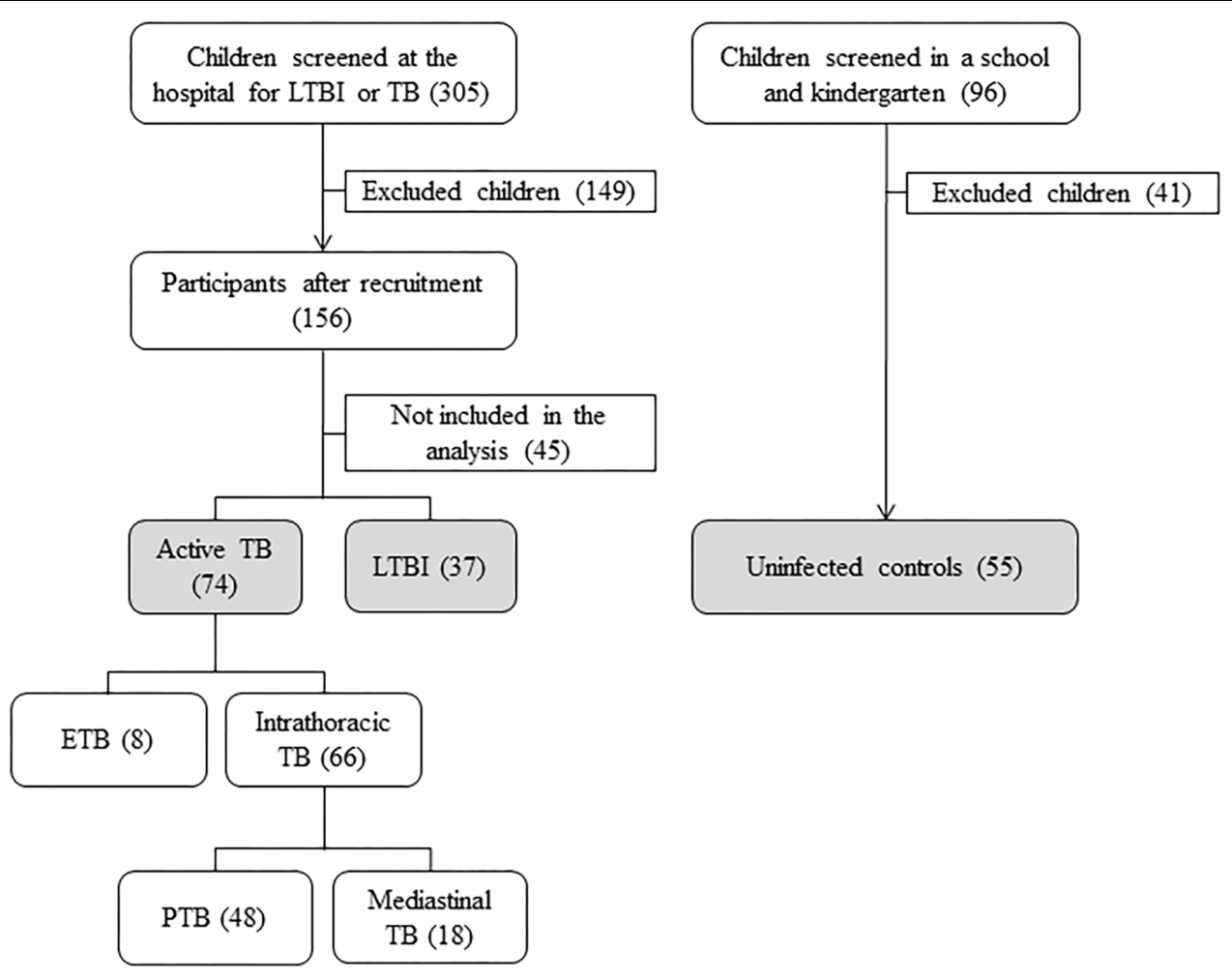

FIGURE 1 | Flow diagram of enrollment. TB, tuberculosis; LTBI, latent tuberculosis infection; ETB, extrathoracic TB; PTB, intrathoracic TB with pulmonary involvement; Mediastinal TB, intrathoracic TB with isolated mediastinal lymphadenopathy in the absence of lung parenchyma involvement.

Furthermore, pairwise comparisons showed that Ag-TB levels of IL-17A and GM-CSF, together with the PHA levels of TNF- $\alpha$ and IL-13, were higher in children with Mediastinal TB than in those with PTB $(p=0.009$ and $0.042, p=0.025$ and $p=0.050$, respectively). Likewise, hemoglobin levels were significantly higher in children with Mediastinal TB than in children with PTB or ETB ( $p=0.014$ and $p=0.014$, respectively). No biomarker showed significant differences between the PTB and ETB groups. Therefore, the groups of children with PTB and extrapulmonary TB were merged and analyzed as a single group (TB, $n=44)$, while children with Mediastinal TB $(n=18)$ were analyzed separately.

Comparisons between the TB group $(n=44)$ and LTBI cases with a positive QFT-GIT ( $n=22$ ) were performed (Table 3 ). The Ag-TB levels of IP-10 along with ferritin levels were significantly higher in children with TB than in children with LTBI $(p=0.005$ and $p=0.019$, respectively). However, the PHA levels of IFN- $\gamma$ were significantly lower in children with TB than in those with LTBI $(p<0.001)$. Although nil levels of IL-5 showed significant differences between groups $(p=0.007)$, this cytokine was not considered in the analysis because almost all the results of the LTBI cases (20/22) and more than half of those of the children who belonged to the TB group (24/44) were below the standard curve. Regarding the association of T. canis with IL-5, no significant differences were found between Ag-TB, PHA and nil IL-5 levels and T. canis in the groups with positive QFT-GIT ( $p$-values of $0.202,0.508$, and 0.053 , respectively). Moreover, the eosinophil count was determined, but because only $25 \%$ of the children had an eosinophil count, this variable was not included in the analysis of the study. Despite the few results, no significant differences were found between Ag-TB, PHA and nil IL-5 levels and eosinophil count in the groups with a positive QFT-GIT ( $p$-values of $0.848,0.923$, and 0.768 , respectively).

\section{Characterization of Subjects With a Negative QFT-GIT Test}

Comparisons among active TB cases $(n=9)$, LTBI cases $(n=12)$ and uninfected controls $(n=55)$ with a negative QFT-GIT $(n=22)$ were performed (Table 4$)$. The PHA levels of IL-10, IL-13, and IL-32, together with the nil levels of TNF- $\alpha$ and IL-10, the underweight levels, and 25(OH)D levels showed significant differences $(p<0.001, p=0.007$, and $p=0.004, p=0.001$ and $p=0.044, p=0.001$, and $p=0.037$, respectively). Furthermore, pairwise comparisons showed that PHA levels of IL-10 were significantly higher in TB and LTBI cases than in uninfected controls $(p=0.020$ and $p=0.001$, respectively), whereas the PHA levels of IL-32 were significantly higher in LTBI cases than in TB cases, and uninfected controls ( $p=0.034$ and $p=0.004$, respectively), and the PHA levels of IL-13 were significantly higher in LTBI cases compared to uninfected controls $(p=0.006)$. Otherwise, the nil TNF- $\alpha$ levels were significantly higher in uninfected controls compared to LTBI cases $(p<0.001)$. Regarding levels of malnourishment, the WAZ $z$-scores were significantly lower in TB and LTBI 
TABLE 1 | Description and comparison of the demographic and clinical characteristics among the study groups according to QFT-GIT results.

Study groups classified by QFT-GIT result $(n=166)$

\begin{tabular}{|c|c|c|c|c|c|c|c|c|}
\hline & \multicolumn{3}{|c|}{ Active TB $(n=74)$} & \multicolumn{3}{|c|}{ LTBI $(n=37)$} & \multirow{2}{*}{$\begin{array}{c}\text { Uninfected }(n=55) \\
\text { Negative } \\
n=55\end{array}$} & \multirow[t]{2}{*}{ p. overall } \\
\hline & $\begin{array}{c}\text { Positive } \\
n=62\end{array}$ & $\begin{array}{c}\text { Negative } \\
n=9\end{array}$ & $\begin{array}{l}\text { Indeterminate } \\
\qquad n=3\end{array}$ & $\begin{array}{c}\text { Positive } \\
n=22\end{array}$ & $\begin{array}{c}\text { Negative } \\
n=12\end{array}$ & $\begin{array}{c}\text { Indeterminate } \\
\qquad n=3\end{array}$ & & \\
\hline Gender & & & & & & & & 0.548 \\
\hline Female & 26 (41.9\%) & 5 (55.6\%) & $2(66.7 \%)$ & $12(54.5 \%)$ & $6(50.0 \%)$ & $3(100 \%)$ & 25 (45.5\%) & \\
\hline Male & 36 (58.1\%) & $4(44.4 \%)$ & 1 (33.3\%) & $10(45.5 \%)$ & $6(50.0 \%)$ & $0(0 \%)$ & 30 (54.5\%) & \\
\hline Age in years & $85.3(44.8)$ & $47.3(51.6)$ & $59.0(32.0)^{\mathrm{a}}$ & $114(45.6)^{a}$ & $80.6(44.6)$ & 48.7 (19.5) & 76.5 (35.2) & 0.001 \\
\hline Range & & & & & & & & 0.008 \\
\hline$\leq 5$ years & $25(40.3 \%)$ & 7 (77.8\%) & $2(66.7 \%)$ & $5(22.7 \%)$ & $6(50.0 \%)$ & $3(100 \%)$ & $32(58.2 \%)$ & \\
\hline$>5$ years & 37 (59.7\%) & 2 (22.2\%) & 1 (33.3\%) & 17 (77.3\%) & $6(50.0 \%)$ & $0(0 \%)$ & $23(41.8 \%)$ & \\
\hline \multicolumn{9}{|l|}{ Body weight-for-age } \\
\hline$Z$-score (SD) & $-1.39(1.70)^{\mathrm{a}}$ & $-1.70(1.09)$ & $-1.78(1.06)$ & $-0.66(0.98)$ & $-1.57(1.50)$ & $-0.92(1.23)$ & $-0.30(1.00)^{\mathrm{a}}$ & 0.001 \\
\hline BCG scar & & & & & & & & 0.424 \\
\hline Yes & $41(69.5 \%)$ & 7 (87.5\%) & $2(66.7 \%)$ & 15 (71.4\%) & $9(100 \%)$ & $1(50.0 \%)$ & 39 (72.2\%) & \\
\hline Hemoglobin (g/dl) & $10.4(2.02)$ & $9.89(0.97)$ & $9.57(0.84)$ & $11.2(1.30)$ & $11.0(1.37)$ & $11.0(3.33)$ & $11.5(0.66)$ & 0.457 \\
\hline
\end{tabular}

Categorical variables are expressed as frequencies ( $n$ ) and percentages (\%), and quantitative variables are expressed as median and interquartile ranges (IQR) or standard deviation (SD). Statistical differences between pairwise comparisons (a). QFT-GIT, QuantiFERON-TB Gold In-Tube; TB, tuberculosis; LTBI, latent tuberculosis infection; Z-score, default classification system used to present child nutritional status; BCG, Bacillus Calmette-Guérin. Bold values, statistical significative values with a $p$-value under 0.05 . 
TABLE 2 | Description and comparison of demographic, clinical, and cytokine responses among active TB cases with positive QFT-GIT.

\begin{tabular}{|c|c|c|c|c|}
\hline \multicolumn{5}{|c|}{ Study subjects with active TB and a positive QFT-GIT assay classified by disease location ( $n=62)$} \\
\hline & $n=36$ & $n=8$ & $n=18$ & \\
\hline Gender & & & & 0.935 \\
\hline Male & $20(55.6 \%)$ & $5(62.5 \%)$ & $11(61.1 \%)$ & \\
\hline Age in years & $76.0[34.5 ; 115]$ & $89.5[55.5 ; 100]$ & $99.0[70.2 ; 130]$ & 0.187 \\
\hline$>5$ years & $19(52.8 \%)$ & $5(62.5 \%)$ & $13(72.2 \%)$ & \\
\hline \multicolumn{5}{|l|}{ Body weight-for-age } \\
\hline Z-score & $-1.24[-2.74 ; 0.08]$ & $-2.07[-2.79 ;-1.13]$ & $-0.98[-2.37 ;-0.21]$ & 0.334 \\
\hline BCG scar & & & & 0.854 \\
\hline Yes & $23(69.7 \%)$ & $5(62.5 \%)$ & $13(72.2 \%)$ & \\
\hline Serology Toxocara spp. & & & & 0.099 \\
\hline Negative & $31(86.1 \%)$ & $5(62.5 \%)$ & $11(64.7 \%)$ & \\
\hline Positive & $5(13.9 \%)$ & $3(37.5 \%)$ & $6(35.3 \%)$ & \\
\hline Ferritin (ng/ml) & 98.4 [33.0; 201] & $138[78.5 ; 218]$ & $64.4[47.4 ; 117]$ & 0.276 \\
\hline $25(\mathrm{OH}) \mathrm{D}(\mathrm{ng} / \mathrm{ml})$ & 26.6 [20.6; 34.5] & $30.2[26.3 ; 34.2]$ & $28.6[21.8 ; 31.2]$ & 0.735 \\
\hline Range & & & & 1.000 \\
\hline Normal levels & $27(75.0 \%)$ & $6(75.0 \%)$ & $14(77.8 \%)$ & \\
\hline Deficiency & $9(25.0 \%)$ & $2(25.0 \%)$ & $4(22.2 \%)$ & \\
\hline \multicolumn{5}{|l|}{ Cytokine response (pg/ml) } \\
\hline \multicolumn{5}{|l|}{$\underline{\mathrm{Ag}-\mathrm{TB}}$} \\
\hline IL-13 & $716[315 ; 1350]$ & $1342[534 ; 1876]$ & $1326[984 ; 1755]$ & 0.071 \\
\hline IL-5 & 3.32 [0.00; 7.05] & $0.00[0.00 ; 13.5]$ & $8.59[3.53 ; 25.7]$ & 0.204 \\
\hline IL-32 & $30.1[-122.86 ; 64.1]$ & $59.4[-25.44 ; 396]$ & $77.1[-10.03 ; 360]$ & 0.222 \\
\hline VEGF & $-381.77[-784.77 ;-120.49]$ & $-903.69[-1128.04 ;-499.36]$ & $-468.37[-931.27 ;-173.86]$ & 0.150 \\
\hline \multicolumn{5}{|l|}{ PHA } \\
\hline TNF- $\alpha$ & $4961[2833 ; 8837]^{\mathbf{a}}$ & $4850[3446 ; 7646]$ & $9762[4829 ; 12489]^{\mathbf{a}}$ & 0.027 \\
\hline $\mid \mathrm{P}-10$ & 4718 [2862; 7972] & 5335 [2623; 16865] & $6952[814 ; 19877]$ & 0.840 \\
\hline IL-10 & $-2.79[-11.05 ; 0.00]$ & $0.00[-0.30 ; 0.91]$ & $0.00[-1.99 ; 0.92]$ & 0.071 \\
\hline IFN- $\gamma$ & 1102 [632; 3913] & 1029 [876; 1878] & 1828 [1131; 8723] & 0.272 \\
\hline IL-1RA & 46679 [27310; 80379] & 39813 [17196; 46881] & 82727 [29720; 142991] & 0.311 \\
\hline IL-17A & $39.8[13.4 ; 129]$ & $9.07[6.54 ; 13.1]$ & $19.8[8.22 ; 81.8]$ & 0.057 \\
\hline GM-CSF & $65.2[34.1 ; 118]$ & $26.7[9.59 ; 46.5]^{\mathbf{b}}$ & $74.7[54.8 ; 112]^{\mathbf{b}}$ & 0.040 \\
\hline IL-13 & $1211[664 ; 1970]^{\mathbf{a}}$ & 1536 [1132; 2023] & $2150[1423 ; 2608]^{\mathbf{a}}$ & 0.042 \\
\hline IL-5 & $4.28[0.00 ; 7.38]$ & $0.00[0.00 ; 0.00]$ & $2.98[0.52 ; 4.76]$ & 0.064 \\
\hline IL-32 & $43.9[-74.11 ; 243]$ & $7.99[-98.12 ; 686]$ & $243[69.0 ; 739]$ & 0.109 \\
\hline VEGF & $-62.75[-368.65 ; 228]$ & $-434.83[-559.52 ;-156.10]$ & $-73.02[-503.76 ; 517]$ & 0.297 \\
\hline \multicolumn{5}{|l|}{ Nil } \\
\hline TNF- $\alpha$ & $151[40.3 ; 250]$ & $33.6[17.3 ; 178]$ & $106[55.3 ; 209]$ & 0.388 \\
\hline IP-10 & $673[412 ; 1220]$ & 745 [529; 1082] & 973 [616; 1201] & 0.573 \\
\hline
\end{tabular}


TABLE 2 | Continued

\begin{tabular}{|c|c|c|c|c|}
\hline \multicolumn{5}{|c|}{ Study subjects with active TB and a positive QFT-GIT assay classified by disease location $(n=62)$} \\
\hline & $n=36$ & $n=8$ & $n=18$ & \\
\hline IL-10 & $8.52[1.62 ; 16.3]$ & $0.88[0.00 ; 5.10]$ & $5.45[3.49 ; 9.86]$ & 0.168 \\
\hline IFN- $\gamma$ & $197[114 ; 509]$ & $310[106 ; 454]$ & $200[171 ; 451]$ & 0.872 \\
\hline IL-1RA & 14867 [9160; 22252] & $11778[6783 ; 27489]$ & $12406[8385 ; 20155]$ & 0.765 \\
\hline IL-13 & $311[147 ; 622]$ & $257[0.00 ; 423]$ & $341[292 ; 444]$ & 0.604 \\
\hline IL-5 & 0.00 [0.00; 5.88] & 0.00 [0.00; 8.95] & 3.26 [0.00; 5.52] & 0.937 \\
\hline IL-32 & 1096 [508; 2241] & 1606 [699; 2579] & 1558 [1101; 2840] & 0.376 \\
\hline VEGF & $702[175 ; 1801]$ & 935 [726; 1128] & 693 [368; 1323] & 0.830 \\
\hline
\end{tabular}

Categorical variables are expressed as frequencies ( $n$ ) and percentages (\%), and quantitative variables are expressed as median and interquartile ranges (IQR) or standard deviation (SD). Statistical differences between pairwise comparisons (a, b). QFT-GIT, QuantiFERON-TB Gold In-Tube; TB, tuberculosis; PTB, Intrathoracic TB with pulmonary involvement; ETB, Extrathoracic TB; Mediastinal TB, Intrathoracic TB with isolated mediastinal lymphadenopathy in the absence of lung parenchyma involvement; Z-score, default classification system used to present child nutritional status; BCG, Bacillus Calmette-Guérin; 25(OH)D, 25-hydroxyvitamin D; Ag-TB, antigendependent response; PHA, mitogen-induced response. Bold values, statistical significative values with a p-value under 0.05 .

TABLE 3 | Description and comparison of demographic, clinical, and cytokine response between groups with a positive QFT-GIT.

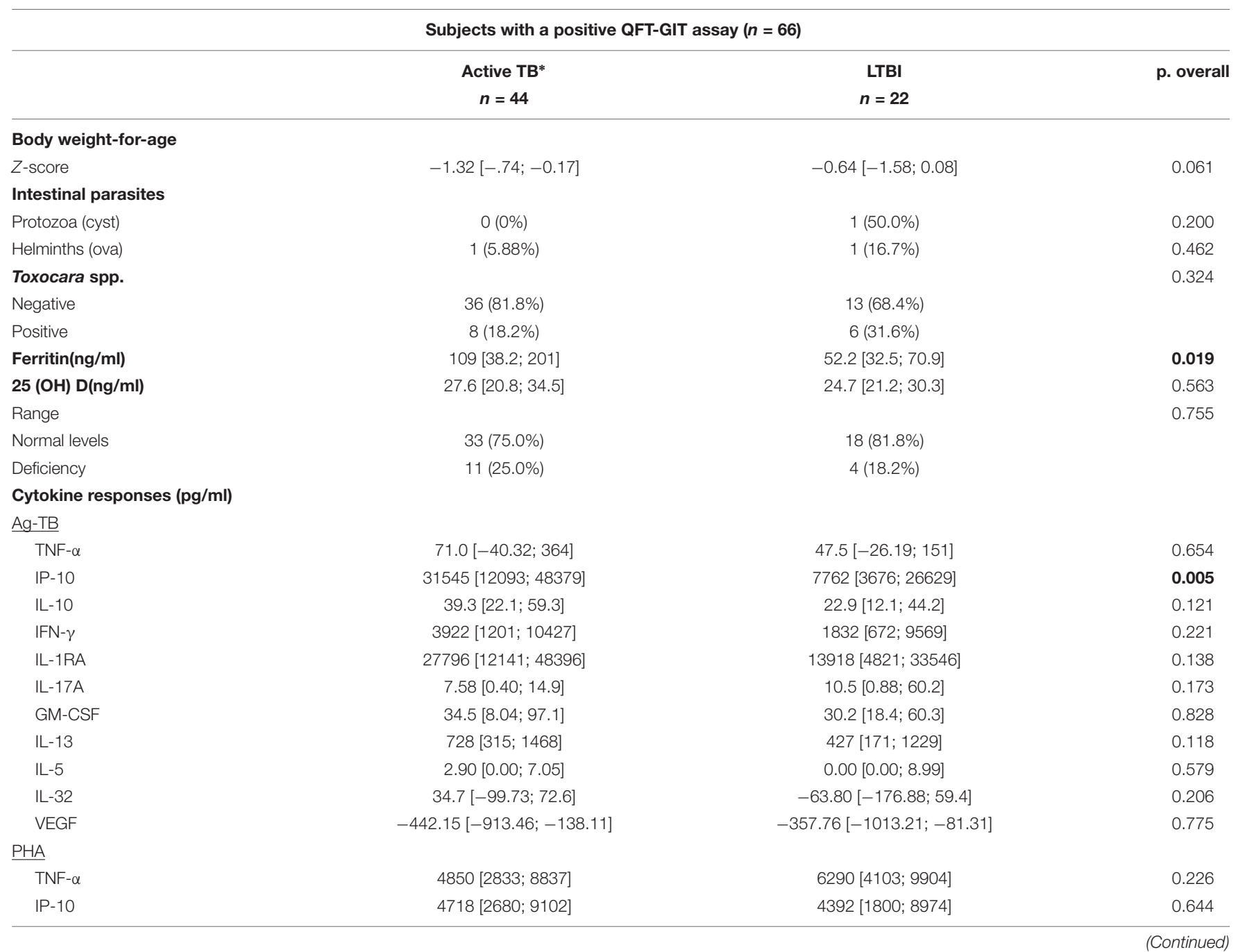




\begin{tabular}{|c|c|c|c|}
\hline \multicolumn{4}{|c|}{ Subjects with a positive QFT-GIT assay ( $n=66$ ) } \\
\hline & $n=44$ & $n=22$ & \\
\hline IL-10 & $-0.47[-9.25 ; 0.00]$ & $-5.14[-8.32 ;-1.05]$ & 0.307 \\
\hline $\mathrm{IFN}-\gamma$ & $1102[717 ; 2984]$ & $4420[2137 ; 11309]$ & $<0.001$ \\
\hline IL-1RA & $42206[27310 ; 69517]$ & $45310[27013 ; 112972]$ & 0.812 \\
\hline IL-13 & $1232[683 ; 1973]$ & $1180[796 ; 1812]$ & 0.946 \\
\hline IL-5 & $2.92[0.00 ; 6.98]$ & $0.91[0.00 ; 10.1]$ & 0.967 \\
\hline IL-32 & $32.9[-74.11 ; 304]$ & $-1.83[-57.38 ; 75.4]$ & 0.391 \\
\hline VEGF & $-108.91[-462.16 ; 181]$ & $-315.24[-871.29 ;-16.37]$ & 0.135 \\
\hline \multicolumn{4}{|c|}{ 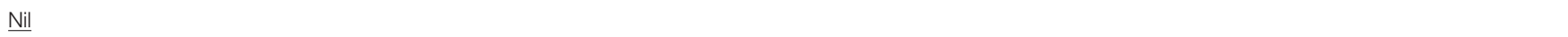 } \\
\hline IL-1RA & $14041[8927 ; 23001]$ & $11090[6497 ; 17128]$ & 0.106 \\
\hline IL-17A & $0.00[0.00 ; 10.4]$ & $0.00[0.00 ; 2.46]$ & 0.764 \\
\hline GM-CSF & $0.89[0.00 ; 12.3]$ & $0.83[0.00 ; 5.30]$ & 0.429 \\
\hline IL-13 & $302[114 ; 595]$ & 256 [146; 499] & 0.683 \\
\hline IL-5 & 0.00 [0.00; 6.96] & 0.00 [0.00; 0.00] & 0.007 \\
\hline IL-32 & 1112 [556; 2241] & 1726 [1033; 2590] & 0.334 \\
\hline VEGF & 809 [209; 1610] & 688 [317; 1381] & 0.870 \\
\hline
\end{tabular}

Categorical variables are expressed as frequencies ( $n$ ) and percentages (\%), and quantitative variables are expressed as median and interquartile ranges (IQR) or standard deviation (SD). QFT-GIT, QuantiFERON-TB Gold In-Tube; TB, tuberculosis; LTBI, latent tuberculosis infection; Z-score, default classification system used to present child nutritional status; BCG, Bacillus Calmette-Guérin; 25(OH)D, 25-hydroxyvitamin D; Ag-TB, antigen-dependent response; PHA, mitogen-induced response. *In this table, children with Mediastinal TB were not included in the active TB cases. Bold values, statistical significative values with a p-value under 0.05 .

cases than in uninfected controls $(p=0.006$ and $p=0.006$, respectively). On the contrary, the median $25(\mathrm{OH}) \mathrm{D}$ levels were significantly higher in LTBI cases compared to uninfected controls $(p=0.022)$.

\section{Discriminative Biomarker Profiles in Subjects With Positive QFT-GIT}

The adjusted logistic model showed a set of 4 biomarkers able to discriminate between active TB and LTBI: ferritin, 25(OH)D, IP-10, and IFN- $\gamma$ (Table 5).

For each increased unit of IFN- $\gamma$ (in Ag-TB responses), IP-10 (in Ag-TB and PHA responses), ferritin, and 25(OH)D, the odds of being classified as a TB case increased by 1.08, 1.80, 1.13, 1.02, and 1.22, respectively. However, for each increased unit of IFN- $\gamma$ (in PHA responses), the odds of being classified as a TB case decreased by 0.46 . Therefore, the profile to classify a subject with TB but not LTBI is that of low IFN- $\gamma$ levels (in PHA responses) but high IP-10 (in Ag-TB and PHA responses), 25(OH)D, and ferritin levels.

The model based on the four host-markers mentioned above was able to correctly classify $93.2 \%$ of children with TB, and $90.0 \%$ of children with LTBI. In addition, the area under the ROC curve of this model was 0.955 (CI 95\%: 0.91 to 1.00), and the positive and negative likelihood ratio were 9.32 and 0.08 , respectively (Figure 2). Furthermore, when Mediastinal TB was included, the model correctly classified $76.2 \%$ of these into the TB group. Supplementary Table 1 summarizes the diagnostic performance of the different combination possibilities of the four host-markers selected in the adjusted logistic model.

\section{Discriminative Biomarker Profiles in Subjects With Negative QFT-GIT}

The adjusted logistic model showed a set of 3 biomarkers able to discriminate among active TB, LTBI, and uninfected controls: IL-10, IL-13, and IL-32 (Table 6). For each increased unit of IL-13 and IL-32 (in PHA responses), the odds of being classified as LTBI case increased by 1.003 and 1.003 , respectively. However, for each increased unit of IL-10 (in PHA responses), the odds of being classified as uninfected control decreased by 0.93 and 0.01 , respectively.

The combination of PHA levels of IL-10, IL-13, and IL-32 detected $11.1 \%$ of active TB cases, $50.0 \%$ of LTBI cases, and $96.4 \%$ of uninfected controls.

\section{DISCUSSION}

This study aimed to identify promising markers to discriminate between active TB and LTBI in children. We obtained a combination based on IP-10, IFN- $\gamma$, ferritin, and 25(OH)D which 
TABLE 4 | Description and comparison of demographic, clinical, and cytokine response between groups with a negative QFT-GIT.

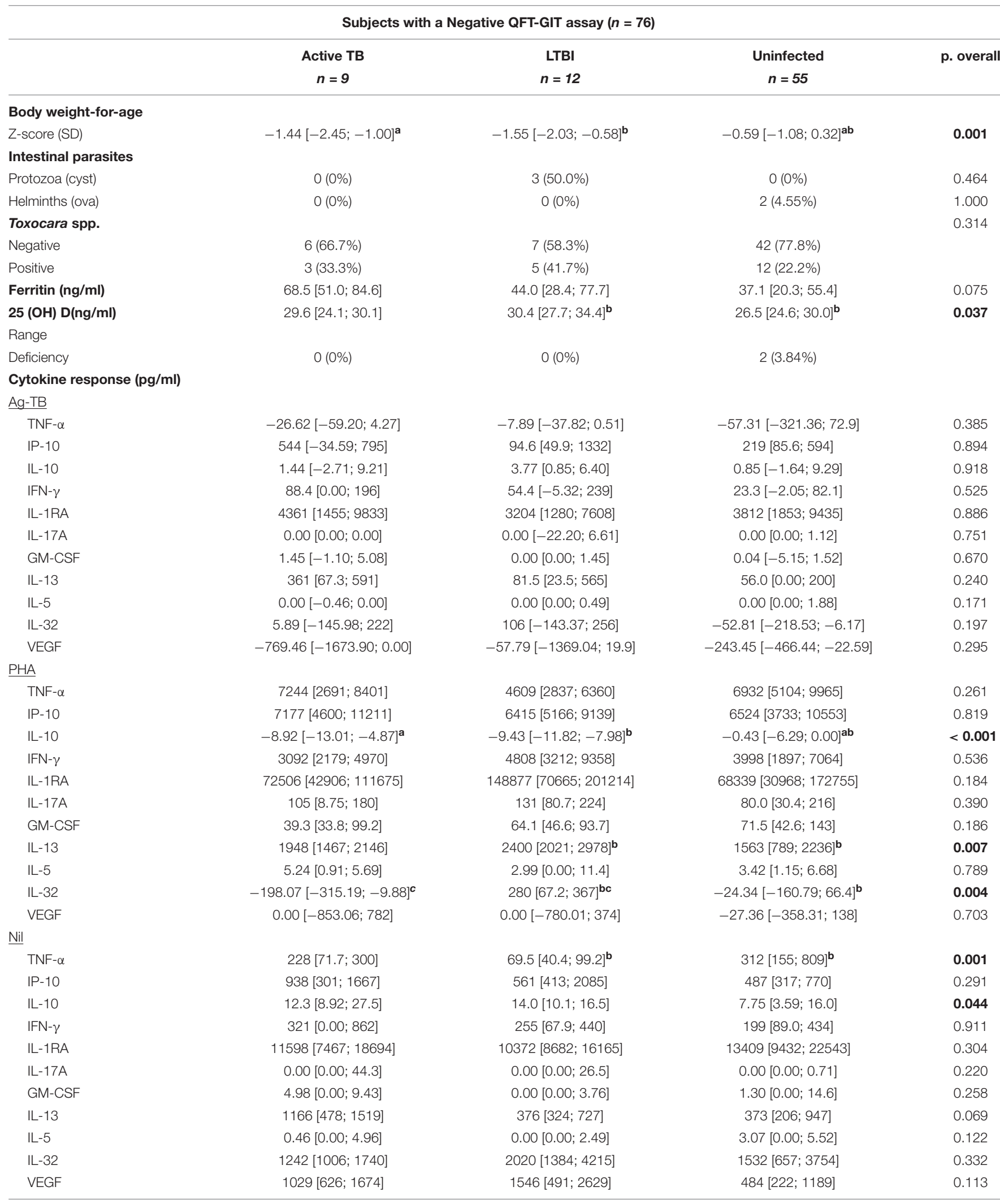

Categorical variables are expressed as frequencies ( $n$ ) and percentages (\%), and quantitative variables are expressed as median and interquartile ranges (IQR) or standard deviation (SD). Statistical differences between pairwise comparisons corrections (a, b, c). QFT-GIT, QuantiFERON-TB Gold In-Tube; TB, tuberculosis; LTBI, latent tuberculosis infection; Z-score, default classification system used to present child nutritional status; BCG, Bacillus Calmette-Guérin; 25(OH)D, 25-hydroxyvitamin D; Ag-TB, antigen-dependent response; PHA, mitogen-induced response. Bold values, statistical significative values with a p-value under 0.05. 
TABLE 5 | Classification table of the model for the study groups with positive QFT-GIT results and variables included in the equation.

Classification table of the Active TB* and LTBI cases with positive QFT-GIT results and variables included in the equation

\begin{tabular}{lccc}
\hline & B & P-value & Odds Ratio [Cl 95\%] \\
\hline $\mathbf{2 5}$ (OH) D (ng/ml) & 0.20 & $\mathbf{0 . 0 1 2}$ & $1.22[1.05 ; 1.43]$ \\
$\begin{array}{l}\text { Ferritin (ng/ml) } \\
\text { Cytokine response (pg/ml) }\end{array}$ & 0.02 & $\mathbf{0 . 0 1 0}$ & $1.02[1.01 ; 1.03]$ \\
Ag-TB & & & \\
\hline IP-10 & & & \\
INF- $\gamma$ & 0.08 & $\mathbf{0 . 0 1 7}$ & $1.08[1.01 ; 1.15]$ \\
PHA & 0.12 & 0.064 & $1.13[0.99 ; 1.29]$ \\
IP-10 & & & \\
INF- $\gamma$ & 0.59 & $\mathbf{0 . 0 0 7}$ & $1.80[1.18 ; 2.75]$ \\
Constant & -0.77 & $\mathbf{0 . 0 0 8}$ & $0.46[0.26 ; 0.82]$ \\
& -8.67 & 0.004 &
\end{tabular}

Binomial Logistic Regression. B, regression coefficient; OR, Odds Ratio; Cl, Confidence Interval. QFT-GIT, QuantiFERON-TB Gold In-Tube; TB, tuberculosis; LTBI, latent tuberculosis infection; 25(OH)D, 25-hydroxyvitamin D; Ag-TB, antigendependent response; PHA, mitogen-induced response. *In this table, children with Mediastinal TB were not included in the active TB cases. ${ }^{\dagger}$ Cytokine levels were multiplied $\times 10$. Bold values, statistical significative values with a $p$-value under 0.05 .

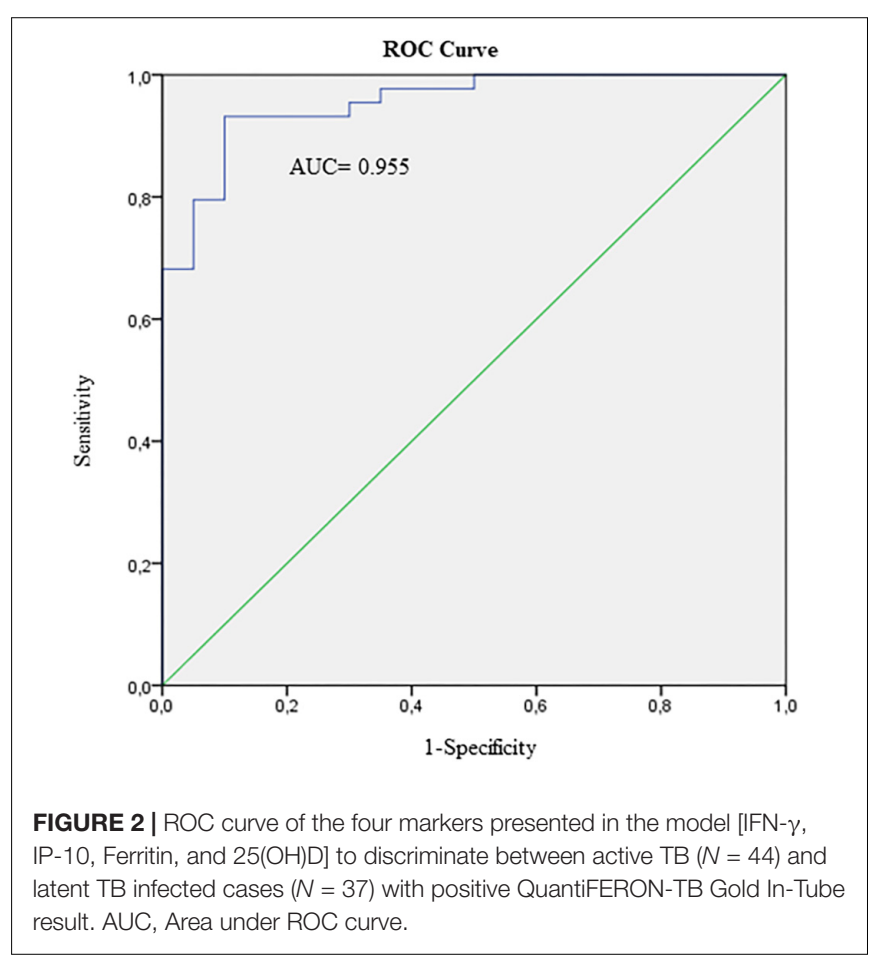

showed diagnostic potential and a predictive value higher than the minimum target product profiles (TPPs) recommended.

\section{Cytokines in M. tuberculosis Infection and Active Disease}

Although IGRAs have become a standard method for the diagnosis of $M$. tuberculosis infection, IFN- $\gamma$ alone is unable to discriminate between TB and LTBI (Denkinger et al., 2015;
TABLE 6 | Classification table for the study groups with negative QFT-GIT results and variables included in the equation.

Classification table of the study groups with negative QFT-GIT results and variables included in the equation

\begin{tabular}{lccc}
\hline Model $^{\mathbf{a}}$ & B & $\boldsymbol{P}$-value & Odds Ratio [Cl 95\%] \\
\hline $\begin{array}{l}\text { Active TB } \\
\text { PHA }(\mathrm{pg} / \mathrm{ml})\end{array}$ & & \\
Constant & -4.67 & & \\
IL-10 & -0.071 & $\mathbf{0 . 0 3 6}$ & $0.93[0.87 ; 0.99]$ \\
IL-13 & $1.25 \times 10^{-3}$ & 0.073 & $1.01[1.00 ; 1.01]$ \\
IL-32 & $3.42 \times 10^{-4}$ & 0.751 & $1.00[0.99 ; 1.01]$ \\
LTBI & & & \\
PHA $(p g / m l)$ & & & \\
Constant & -8.873 & & \\
IL-10 & -0.095 & $\mathbf{0 . 0 0 9}$ & $0.910[0.85 ; 0.98]$ \\
IL-13 & $3.06 \times 10^{-3}$ & $\mathbf{0 . 0 0 1}$ & $1.003[1.001 ; 1.005]$ \\
IL-32 & $2.60 \times 10^{-3}$ & $\mathbf{0 . 0 0 7}$ & $1.003[1.001 ; 1.005]$ \\
\hline
\end{tabular}

aThe reference category is the uninfected control group. Multivariate Logistic Regression. B, regression coefficient; OR, Odds Ratio; Cl, Confidence Interval. QFT-GIT, QuantiFERON-TB Gold In-Tube; TB, tuberculosis; LTBI, latent tuberculosis infection; PHA, mitogen-induced response. Bold values, statistical significative values with a $p$-value under 0.05

Latorre and Domínguez, 2015; Pai and Behr, 2016). Previous studies showed evidence of the low sensitivity of the IGRAs in individuals with a depressed or immature immune system such as immunosuppressed patients or young children (Latorre et al., 2014). In our study, very low Ag-TB cytokine levels were found in subjects with negative QFT-GIT in comparison with those with a positive QFT-GIT. Therefore, no cytokine or combination of cytokines showed potential for the discrimination between uninfected controls and M. tuberculosis-infected children with a negative QFT-GIT result. This finding is in line with Lighter-Fisher et al., who reported that LTBI children under 5 years old showed negative QFT-GIT results and very low levels of cytokines (Lighter-Fisher et al., 2010). These results corroborate the weakened immune response of young children in protecting against $M$. tuberculosis and, therefore, their susceptibility to progress to TB disease. Conversely, in the present study, statistically significant differences were found between markers with potential to diagnose $\mathrm{TB}$ and to discriminate active TB from LTBI in $M$. tuberculosis-infected children with a positive QFT-GIT result.

Since children become more susceptible to developing intrathoracic and ETB disease following exposure to M. tuberculosis, several studies have examined the patterns of expression of a variety of biomarkers to understand the progression of intrathoracic and ETB disease in children (Whittaker et al., 2012). Similar to Kumar et al. (2013) in the present study no marker was able to differentiate between subjects with intrathoracic TB and ETB (Kumar et al., 2013). Although mediastinal TB subjects showed an immune profile similar to that of intrathoracic and ETB, five biomarkers (IL-17, GM-CSF, TNF- $\alpha$, IL-13, and ferritin) showed potential to identify mediastinal TB subjects in the TB group. Radiographic signs of mediastinal TB have been related to the onset of primary TB 
in children (Thomas, 2019). Considering that TB represents a dynamic continuum of states in which the dichotomous distinction between infection and disease is often difficult to differentiate (Perez-Velez et al., 2017), the previous cytokines suggest a potential for detecting $M$. tuberculosis-infected children most likely to progress to the disease and/or early stages of TB.

In subjects with a positive QFT-GIT result, IP-10, IFN- $\gamma$, IL-5, and ferritin responses individually showed statistically significant differences between active TB and LTBI. However, the combination of IP-10, IFN- $\gamma$, ferritin, and 25(OH)D achieved the best diagnostic performance to discriminate between active TB and LTBI cases. IP-10 and IFN- $\gamma$ play an important role in the immune response to $M$. tuberculosis infection (Chegou et al., 2014). Several studies have described the good performance of IP-10 when compared or combined with IFN- $\gamma$ for the diagnosis of TB and the discrimination between TB and LTBI in children, respectively (Ruhwald et al., 2012; Villar-Hernandez et al., 2017). While IP-10 is a chemokine involved in the trafficking and stimulation of monocytes and Th1 cells activated in response to inflammatory foci, IFN- $\gamma$ is essential for the mediation of the adaptive immune response against $M$. tuberculosis (Ruhwald et al., 2008). In contrast to IFN- $\gamma$, IP-10 induces a robust, specific M. tuberculosis response not influenced by age (Ruhwald et al., 2012). Indeed, according to previous studies, IP-10 shows the same kinetics as IFN- $\gamma$ but at levels up to 10 -fold higher (Lighter et al., 2009; Ruhwald et al., 2011).

Previous studies in M. tuberculosis-infected adults and children observed variations in cytokine responses after PHA-stimulation (Mueller et al., 2008; Ruhwald et al., 2008, 2011; Alsleben et al., 2012; Jeong et al., 2015). In our study, PHA levels of IFN- $\gamma$ were significantly higher in LTBI cases compared to TB cases. Similar findings have been observed in M. tuberculosis-infected adults whose blood was stimulated with QFT-GIT peptides (Jeong et al., 2015). Therefore, PHA-induced responses were considered to evaluate host immune status for the discrimination between TB and LTBI in children. On the other hand, in this study, PHA levels of IP-10 were very similar to those obtained in M. tuberculosis-specific stimulation. In this regard, Ruhwald et al. (2008) suggested that PHA was a powerful inducer of IFN- $\gamma$. However, the same was not observed with IP-10, with which PHA levels were rather low.

In our study, IL-5 levels in unstimulated samples showed statistically significant differences between TB and LTBI cases. However, this cytokine was not consistently expressed. Studies in adults have shown the potential of IL-5 for discriminating between TB and LTBI cases (Won et al., 2017). However, the diagnostic value of IL-5 in children is relatively unknown (Armand et al., 2014).

In this study, cytokines levels were measured from supernatants remaining from QFT-GIT tubes. Recently, QuantiFERON-TB ${ }^{\circledR}$ Gold Plus (QFT-Plus), has been introduced in the new generation of QFT assay. For this new test there are two TB-specific antigen tubes, called TB1 and TB2. The TB1 tube, contains long peptides derived from ESAT-6 and CFP-10 (for this new test the peptide TB-7.7 has been removed), and it is designed to induce a specific CD4 T cells response. TB2 contains both the same long peptides of TB1 and newly designed shorter peptides to induce interferon (IFN)-g production by both CD4 and CD8 T-cells (Barcellini et al., 2016). The determination of these biomarkers could be performed using the TB1 tube from the QFT-Plus assay, since it contains almost the same relatively long peptides from $M$. tuberculosis antigens (ESAT-6 y CFP-10) to mainly stimulate CD4 + T cells as those used in the QFT-GIT assay antigen tube. Although QFT-Plus does not contain the TB7.7 antigens, it has been shown that the absence of the TB7.7 antigen from the QFT-Plus does not significantly impact assay performance (Theel et al., 2018).

\section{Individual Factors in M. tuberculosis Infection and Disease}

The mechanism by which host, pathogens and extrinsic factors interact as final determinants of disease outcome and TB transmission is an active area of research (Bastos et al., 2018). In this context, we characterized a series of individual factors, among which ferritin and $25(\mathrm{OH}) \mathrm{D}$ were markers that showed potential for the discrimination between active TB and LTBI in children.

Iron is an essential cofactor for mycobacteria propagation during infection (Olakanmi et al., 2000), and successful protective host-immune response (Mainou-Fowler and Brock, 1985). It is known that acute phase proteins stimulate or inhibit their production in response to inflammatory processes such as infections. In our study, ferritin levels in LTBI cases were lower than in active TB cases. Previous findings by our group showed lower ferritin levels in children with a positive QFTGIT at the onset of $M$. tuberculosis infection (Pérez-Porcuna et al., 2014). Similar studies showed higher median ferritin levels in TB adults than in the other study groups (Jacobs et al., 2016). A possible explanation for this result is that ferritin is a recognized acute phase protein in iron storage processes and is closely linked to host response in M. tuberculosis (Thom et al., 2012).

In the present study, no statistically significant differences were found in $25(\mathrm{OH}) \mathrm{D}$ alone between study groups. Vitamin $\mathrm{D}$ is a steroid hormone with pleiotropic actions in many body tissues and cells, including cells of the immune system (Nair et al., 2018). Several studies suggest that $25(\mathrm{OH}) \mathrm{D}$ deficiency (below $20 \mathrm{ng} / \mathrm{ml}$ ) could compromise antibacterial activity and increase the risk of TB disease by preventing the initiation of immune response mediated by vitamin D (Hewison, 2012).

The analysis of $25(\mathrm{OH}) \mathrm{D}$ in combination with IP-10, IFN- $\gamma$ and ferritin was found to be useful to discriminate between active TB and LTBI cases. We observed an inverse correlation between the $25(\mathrm{OH}) \mathrm{D}$ and PHA levels of IFN$\gamma$ in active $\mathrm{TB}$ cases in comparison to LTBI cases. In vitro studies have demonstrated how vitamin $\mathrm{D}$ induces innate antimicrobial responses and suppresses proinflammatory cytokine responses (Coussens et al., 2012). Likewise, Ragab et al. (2016) observed a negative association between the addition of vitamin D and PHA-induced IFN- $\gamma$ levels (Nonnecke et al., 2003) suggesting the multiple mechanisms in which vitamin $\mathrm{D}$ is involved in the protection of the host against M. tuberculosis infection. 


\section{Promising Biomarker-Based Diagnostic Tests in Children}

A recent systematic review by Togun et al. (2018) evaluated biomarkers able to diagnose $\mathrm{TB}$ in children. All the studies performed in QFT-GIT supernatants presented at least a combination of two markers for the discrimination of active TB and LTBI in children, demonstrating the involvement of several mechanisms mediated by different host markers in M. tuberculosis infection and disease (Togun et al., 2018).

Several studies have suggested that IL- 2 and TNF- $\alpha$ may be reliable cytokines for the discrimination between active TB and LTBI cases in children (Lighter-Fisher et al., 2010; Gourgouillon et al., 2012). However, we did not find any significant differences in TNF- $\alpha$, and IL-2 responses in unstimulated and stimulated samples were lower than the minimum concentrations detected in the standard curve of the experiment. Although our findings may not coincide with some mainly previously cited observations, direct comparisons of biomarkers among studies in children are complex due to population variability, the burden of $M$. tuberculosis presented in the different settings, and age variations that could affect the state of maturity of the immune response of the child.

Regarding the possible use of biomarkers in the field, considering the TPPs recommended by FIND/WHO, the sensitivity and specificity achieved by the combination of IFN- $\gamma$, IP-10, ferritin and 25(OH)D were optimal (Denkinger et al., 2015). In recent years, two case-control studies presented two models for the diagnosis of TB in children with minimal TPPs higher than those reached in this study for the development of a new diagnostic test (Armand et al., 2014; Zhou et al., 2017). However, they were not able to discriminate between active TB and LTBI cases. In our study, the combination of IP-10, IFN- $\gamma$, ferritin, and 25(OH)D achieved the best diagnostic performance with correct classification of active TB cases (93.2\%) and LTBI cases (90.0\%). Moreover, this combination of biomarkers correctly classified $76.2 \%$ of the mediastinal TB subjects. Lastly, this combination could be a good diagnostic test to confirm active $\mathrm{TB}(\mathrm{LR}+=9.32)$ and a very robust test to rule out cases with active TB from cases with LTBI $(\mathrm{LR}-=0.08)$.

\section{Limitations and Strengths of the Study}

One limitation of the studies evaluating diagnostic tests for TB in children is the lack of a microbiological gold standard and adequate, validated clinical scoring systems, resulting in low diagnostic sensitivity in children. In this study, TST was defined as a criterion for inclusion of the screened TB and LTBI children to improve patient classification. However, the inclusion criterion mentioned above may have affected the specificity of diagnosis of some risk groups with a vulnerable immune system including malnourished children. Despite attempting to control most of the risk factors, the vulnerability of the study population and context did not allow evaluation of some factors which may affect the immune system (such as cytomegalovirus and allergies). An important strength of this study is the strict criteria for the classification of the study groups (Graham et al., 2015). It should be noted that in this case-control study, the sample size, age ranges according to sex and nutritional status were widely represented. However, according to the results obtained, additional studies are needed to validate the performance of the current model in children with other respiratory infections. The identification of these biomarkers has the same technical difficulty as performance of the QFT-GIT assay. However, the combination of biomarkers presented in this study might also have a great potential to discriminate between TB and LTBI in children. To this end, future studies should be conducted validating the identification of the biomarkers proposed in this study in other geographical areas and different populations.

\section{CONCLUSION}

Our findings suggest the diagnostic potential of the combination of IFN- $\gamma$, IP-10, ferritin and 25(OH)D detected in supernatants in QFT-GIT tubes for the diagnosis of pediatric TB and discrimination between TB and LTBI. In addition, these markers may be useful for the identification of the onset of primary $\mathrm{TB}$, although future investigations in transversal and prospective longitudinal studies are warranted. This study highlights potential markers with optimal diagnostic accuracy for improving the management of TB diagnosis in children. Finally, developing a rapid diagnostic test based on the immunological biomarkers studied would improve the access of $\mathrm{TB}$ services, thereby promoting early diagnosis of pediatric TB and LTBI.

\section{ETHICS STATEMENT}

This study was approved by the Health Research Ethics Committee of the University of Barcelona and the Haitian National Ethics Committee (project number IRB00003099). Before participation, written informed consent was obtained from the child's parents or guardian.

\section{AUTHOR CONTRIBUTIONS}

PC-d-B, CA, TP-P, RA, and JD conceived and designed the study. PC-d-B, MJC, MN, and JG collected the data. PC-d-B, RV-H, RA, TP-P, IL, LCA, BSM, and JD analyzed and interpreted the data. PC-d-B, TP-P, RA, JD, and RV-H drafted the article. All authors contributed toward data analysis, drafting and revising the paper and agree to be accountable for all aspects of the work.

\section{FUNDING}

This work was supported by grants from: (i) the Isolana Foundation, (ii) the Maria Francisca de Roviralta Foundation 
expedient AT/MA 1-19/07/2017, AT/MA 70-27/04/2016, and AT/MA 3-22/10/2014, (iii) the Instituto de Salud Carlos III (PI-16/1912), integrated in the Plan Nacional de I+D+I and cofounded by the ISCIII-Subdirección General de Evaluación and the Fondo Europeo de Desarrollo Regional (FEDER), (iv) CERCA Program/Generalitat de Catalunya, (v) el Departament de Salut de la Generalitat de Catalunya, en la convocatòria corresponent a l'any 2017 de concessió de subvencions del Pla Estratègic de Recerca i Innovació en Salut (PERIS) 2016-2020 modalitat intesificació professionals de salut expedient SLT006/17/144, and (vi) Fundació Recerca i Docència Mútua Terrassa.

\section{ACKNOWLEDGMENTS}

We thank the staff (especially Mr. Louis Yves and Ms. Achil Nancy, nurses and laboratory technicians) of the pediatric Hospital Saint Damien of the Nos Petits Frères et Soeurs

\section{REFERENCES}

Ahmadi, N. A., and Damraj, F. A. (2009). A field evaluation of formalin-gasoline technique in the concentration of stool for detection of intestinal parasites. Parasitol. Res. 104, 553-557. doi: 10.1007/s00436-008-1229-1224

Alsleben, N., Ruhwald, M., Rüssmann, H., Marx, F. M., Wahn, U., and Magdorf, K. (2012). Interferon-gamma inducible protein 10 as a biomarker for active tuberculosis and latent tuberculosis infection in children: a case-control study. Scand. J. Infect. Dis. 44, 256-262. doi: 10.3109/00365548.2011.632644

American Academy of Pediatrics Tuberculosis (2009). Red Book: 2009 Report of the Committee on Infectious Diseases, 28th Edn. Elk Grove Village, IL: AMITA Health Medical Group.

Armand, M., Chhor, V., de Lauzanne, A., Guérin-El Khourouj, V., Pédron, B., Jeljeli, M., et al. (2014). Cytokine responses to quantiferon peptides in pediatric tuberculosis: a pilot study. J. Infect. 68, 62-70. doi: 10.1016/j.jinf.2013.08.005

Augustynowicz-Kopeć, E., Jagielski, T., Kozińska, M., Kremer, K., van Soolingen, D., Bielecki, J., et al. (2012). Transmission of tuberculosis within familyhouseholds. J. Infect. 64, 596-608. doi: 10.1016/j.jinf.2011.12.022

Barcellini, L., Borroni, E., Brown, J., Brunetti, E., Codecasa, L., Cugnata, F., et al. (2016). First independent evaluation of QuantiFERON-TB Plus performance. Eur. Respir. J. 47, 1587-1590. doi: 10.1183/13993003.02033-2015

Bass, J. R., Farer, L. S., Hopewell, P. C., Jacobs, R. F., and Snider, D. E. Jr. (1990). American thoracic society. diagnostic standards and classification of tuberculosis. Am. Rev. Respir. Dis. 142, 725-735. doi: 10.1164/ajrccm/142.3.725

Bastos, H. N., Osório, N. S., Gagneux, S., Comas, I., and Saraiva, M. (2018). The troika host-pathogen-extrinsic factors in tuberculosis: modulating inflammation and clinical outcomes. Front. Immunol. 8:1948. doi: 10.3389/ fimmu.2017.01948

Chegou, N. N., Heyckendorf, J., Walzl, G., Lange, C., and Ruhwald, M. (2014). Beyond the IFN- $\gamma$ horizon: biomarkers for immunodiagnosis of infection with mycobacterium tuberculosis. Eur. Respir. J. 43, 1472-1486. doi: 10.1183/ 09031936.00151413

Coussens, A. K., Wilkinson, R. J., Hanifa, Y., Nikolayevskyy, V., Elkington, P. T., Islam, K., et al. (2012). Vitamin D accelerates resolution of inflammatory responses during tuberculosis treatment. Proc. Natl. Acad. Sci. U.S.A. 109, 15449-15454. doi: 10.1073/pnas.1200072109

Denkinger, C. M., Kik, S. V., Cirillo, D. M., Casenghi, M., Shinnick, T., Weyer, K., et al. (2015). Defining the needs for next generation assays for tuberculosis. J. Infect. Dis. 211, S29-S38. doi: 10.1093/infdis/jiu821

Dodd, P. J., Yuen, C. M., Becerra, M. C., Revill, P., Jenkins, H. E., and Seddon, J. A. (2018). Potential effect of household contact management on childhood tuberculosis: a mathematical modelling study. Lancet Glob. Heal. 6, e1329-e1338. doi: 10.1016/S2214-109X(18)30401-7
Foundation, Port-au-Prince, for their unconditional support throughout this research project, and for valuable assistance and care in recruiting the patients for this study. We thank Mr. Movil Junior Wilfrid of the Ministère de la Santé Publique d'Haiti for his help during the household screening of children with a household $\mathrm{TB}$ contact. Finally, we also thank Dr. Jaume Ollé Goig from the ACTMON Association for his support to the TB unit of the Saint Damien Hospital that had an indirect positive influence on this project. The funders were not involved in any of the stages of the study, from its design to submission of the manuscript for publication.

\section{SUPPLEMENTARY MATERIAL}

The Supplementary Material for this article can be found online at: https://www.frontiersin.org/articles/10.3389/fmicb. 2019.01855/full\#supplementary-material

Dodd, P. J., Yuen, C. M., Sismanidis, C., Seddon, J. A., and Jenkins, H. E. (2017). The global burden of tuberculosis mortality in children: a mathematical modelling study. Lancet Glob. Heal. 5, e898-e906. doi: 10.1016/S2214-109X(17) 30289-89

Dominguez, J., Latorre, I., Altet, N., Mateo, L., De Souza-Galvao, M., RuizManzano, J., et al. (2009). IFN-gamma-release assays to diagnose TB infection in the immunocompromised individual. Expert Rev. Respir. Med. 3, 309-327. doi: 10.1586/ers.09.20

Gourgouillon, N., De Lauzanne, A., Cottart, C. H., Curis, E., Debord, C., Guérin-El Khourouj, V., et al. (2012). TNF- $\alpha / \mathrm{IL}-2$ ratio discriminates latent from active tuberculosis in immunocompetent children: a pilot study. Pediatr. Res. 72, 370-374. doi: 10.1038/pr.2012.89

Graham, S. M., Cuevas, L. E., Jean-Philippe, P., Browning, R., Casenghi, M., Detjen, A. K., et al. (2015). Clinical case definitions for classification of intrathoracic tuberculosis in children: an update. Clin. Infect. Dis. 61, S179-S187. doi: 10. 1093/cid/civ581

Hewison, M. (2012). An update on vitamin D and human immunity. Clin. Endocrinol. 76, 315-325. doi: 10.1111/j.1365-2265.2011. 04261.x

Ibrahim, M. K., Zambruni, M., Melby, C. L., and Melby, P. C. (2017). Impact of childhood malnutrition on host defense and infection. Clin. Microbiol. Rev. 30, 919-971. doi: 10.1128/CMR.00119-6

Jacobs, R., Maasdorp, E., Malherbe, S., Loxton, A. G., Stanley, K., Van Der Spuy, G., et al. (2016). Diagnostic potential of novel salivary host biomarkers as candidates for the immunological diagnosis of tuberculosis disease and monitoring of tuberculosis treatment response. PLoS One 11:e0160546.doi: 10.1371/journal.pone.01 60546

Jeong, Y. H., Hur, Y. G., Lee, H., Kim, S., Cho, J. E., Chang, J., et al. (2015). Discrimination between active and latent tuberculosis based on ratio of antigenspecific to mitogen-induced IP-10 production. J. Clin. Microbiol. 53, 504-510. doi: 10.1128/JCM.02758-4

Katz, N., Chaves, A., and Pellegrino, J. (1972). A simple device for quantitative stool thick-smear technique in schistomiasismansoni. Rev. Med. Trop. São Paulo 14, 397-400.

Kumar, N. P., Anuradha, R., Andrade, B. B., Suresh, N., Ganesh, R., Shankar, J., et al. (2013). Circulating biomarkers of pulmonary and extrapulmonary tuberculosis in children. Clin. Vaccine Immunol. 20, 704-711. doi: 10.1128/CVI. 00038-13

Kyu, H. H., Maddison, E. R., Henry, N. J., Mumford, J. E., Barber, R., Shields, C., et al. (2018). The global burden of tuberculosis: results from the global burden of disease study 2015. Lancet Infect. Dis. 18, 261-284. doi: 10.1016/S1473-3099(17) 30703-X 
Latorre, I., Díaz, J., Mialdea, I., Serra-Vidal, M., Altet, N., Prat, C., et al. (2014). IP-10 is an accurate biomarker for the diagnosis of tuberculosis in children. J. Infect. 69, 590-599. doi: 10.1016/j.jinf.2014.06.013

Latorre, I., and Domínguez, J. (2015). Dormancy antigens as biomarkers of latent tuberculosis infection. EBIOM 2, 790-791. doi: 10.1016/j.ebiom.2015.06.017

Lighter, J., Rigaud, M., Huie, M., Peng, C. H., and Pollack, H. (2009). Chemokine IP-10: an adjunct marker for latent tuberculosis infection in children. Int. J. Tuberc. Lung Dis. 13, 731-736.

Lighter-Fisher, J., Peng, C. H., and Tse, D. B. (2010). Cytokine responses to QuantiFERON§peptides, purified protein derivative and recombinant ESAT-6 in children with tuberculosis. Int. J. Tuberc. Lung Dis. 14, 1548-1555.

Mainou-Fowler, T., and Brock, J. H. (1985). Effect of iron deficiency on the response of mouse lymphocytes to concanavalin a: the importance of transferrin-bound iron. Immunology 54, 325-332.

Marais, B. J., Gie, R. P., Schaaf, H. S., Hesseling, A. C., Obihara, C. C., Nelson, L. J., et al. (2004). The clinical epidemiology of childhood pulmonary tuberculosis: a critical review of literature from the pre-chemotherapy era. Int. J. Tuberc. Lung Dis. 8, 278-285.

Michael, F., and Holick, M. (2007). Vitamin D deficiency. N. Engl. J. Med. 357, 266-281.

Mueller, H., Detjen, A. K., Schuck, S. D., Gutschmidt, A., Wahn, U., Magdorf, K., et al. (2008). Mycobacterium tuberculosis-specific CD4+, IFN $\gamma+$, and TNF $\alpha+$ multifunctional memory $\mathrm{T}$ cells coexpress GM-CSF. Cytokine 43, 143-148. doi: 10.1016/j.cyto.2008.05.002

Nair, P., Venkatesh, B., and Center, J. R. (2018). Vitamin D deficiency and supplementation in critical illness-the known knowns and known unknowns. Crit. Care 22, 1-9. doi: 10.1186/s13054-018-2185-88

Nicol, M. P., Gnanashanmugam, D., Browning, R., Click, E. S., Cuevas, L. E., Detjen, A., et al. (2015). A blueprint to address research gaps in the development of biomarkers for pediatric tuberculosis. Clin. Infect. Dis. 61, S164-S172. doi: 10.1093/cid/civ613

Nonnecke, B. J., Waters, W. R., Foote, M. R., Horst, R. L., Fowler, M. A., and Miller, B. L. (2003). In vitro effects of 1, 25-dihydroxyvitamin D 3 on interferon- $\gamma$ and tumor necrosis factor- $\alpha$ secretion by blood leukocytes from young and adult cattle Vaccinated with mycobacterium bovis BCG. Int. J. Vitam. Nutr. Res. 73, 235-244. doi: 10.1024//0300-9831.73.4.235

Olakanmi, O., Britigan, B. E., and Schlesinger, L. S. (2000). Gallium disrupts iron metabolism of mycobacteria residing within human macrophages. Infect. Immun. 68, 5619-5627. doi: 10.1128/IAI.68.10.5619-5627.2000

Pai, M., and Behr, M. (2016). Latent mycobacterium tuberculosis infection and interferon-gamma release assays. Microbiol. Spectr. 4, 1-10. doi: 10.1128/ microbiolspec.TBTB2-0023-16

Pérez-Porcuna, T. M., Ascaso, C., Malheiro, A., Abellana, R., Martins, M., Sardinha, J. F. J., et al. (2014). Mycobacterium tuberculosis infection in young children: analyzing the performance of the diagnostic tests. PLoS One 9:e97992. doi: 10.1371/journal.pone.0097992

Perez-Velez, C. M., Roya-Pabon, C. L., and Marais, B. J. (2017). A systematic approach to diagnosing intra-thoracic tuberculosis in children. J. Infect. 74, S74-S83. doi: 10.1016/S0163-4453(17)30195-90

Ragab, D., Soliman, D., Samaha, D., and Yassin, A. (2016). Vitamin D status and its modulatory effect on interferon gamma and interleukin-10 production by peripheral blood mononuclear cells in culture. Cytokine 85, 5-10. doi: 10.1016/ j.cyto.2016.05.024

Ruhwald, M., Aabye, M. G., and Ravn, P. (2012). IP-10 release assays in the diagnosis of tuberculosis infection: current status and future directions. Expert Rev. Mol. Diagn. 12, 175-187. doi: 10.1586/erm.11.97

Ruhwald, M., Bodmer, T., Maier, C., Jepsen, M., Haaland, M. B., Eugen-Olsen, J., et al. (2008). Evaluating the potential of IP-10 and MCP-2 as biomarkers for the diagnosis of tuberculosis. Eur. Respir. J. 32, 1607-1615. doi: 10.1183/09031936. 00055508

Ruhwald, M., Dominguez, J., Latorre, I., Losi, M., Richeldi, L., Pasticci, M. B., et al. (2011). A multicentre evaluation of the accuracy and performance of IP-10 for the diagnosis of infection with M. tuberculosis. Tuberculosis 91, 260-267. doi: 10.1016/j.tube.2011.01.001

Theel, E. S., Hilgart, H., Breen-Lyles, M., McCoy, K., Flury, R., Breeher, L. E., et al. (2018). Comparison of the QuantiFERON-TB gold plus and QuantiFERON-TB gold in-tube interferon gamma release assays in patients at risk for tuberculosis and in health care workers. J. Clin. Microbiol. 56, 1-12. doi: 10.1128/JCM. 00614-8

Thom, R. E., Elmore, M. J., Williams, A., Andrews, S. C., Drobniewski, F., Marsh, P. D., et al. (2012). The expression of ferritin, lactoferrin, transferrin receptor and solute carrier family $11 \mathrm{~A} 1$ in the host response to BCG-vaccination and mycobacterium tuberculosis challenge. Vaccine 30, 3159-3168. doi: 10.1016/j. vaccine. 2012.03 .008

Thomas, T. A. (2019). Tuberculosis in children. Thorac. Surg. Clin. 29, 109-121. doi: 10.1016/j.thorsurg.2018.09.009

Togun, T. O., MacLean, E., Kampmann, B., and Pai, M. (2018). Biomarkers for diagnosis of childhood tuberculosis: a systematic review. PLoS One 13, 1-19. doi: 10.1371/journal.pone.0204029

Velasco-Arnaiz, E., Soriano-Arandes, A., Latorre, I., Altet, N., Dominguez, J., Fortuny, C., et al. (2018). Performance of tuberculin skin tests and interferongamma release assays in children younger than 5 years. Pediatr. Infect. Dis. J. 37, 1235-1241. doi: 10.1097/INF.0000000000002015

Villar-Hernandez, R., Latorre, I., Minguez, S., Diaz, J., Garcia-Garcia, E., MurielMoreno, B., et al. (2017). Use of IFN-gamma and IP-10 detection in the diagnosis of latent tuberculosis infection in patients with inflammatory rheumatic diseases. J. Infect. 75, 315-325. doi: 10.1016/j.jinf.2017.07.004

Whittaker, E., Jones, C., and Kampmann, B. (2012). TB in children. Eur. Respir. Monogr. 58, 206-218.

Won, E. J., Choi, J. H., Cho, Y. N., Jin, H. M., Kee, H. J., Park, Y. W., et al. (2017). Biomarkers for discrimination between latent tuberculosis infection and active tuberculosis disease. J. Infect. 74, 281-293. doi: 10.1016/j.jinf.2016.11.010

World Health Organization [WHO] (2009). AnthroPlus for Personal Computers Manual: Software for Assessing Growth of the World's Children and Adolescents. Geneva: World Health Organization.

World Health Organization [WHO] (2013). Guideline: Updates on the Management of Severe Acute Malnutrition in Infants and Children. Geneva: World Health Organization.

World Health Organization (2017). Consensus Meeting Report: Development of a Target Product Profile (TPP) and a Framework for Evaluation for a Test for Predicting Progression from Tuberculosis Infection to Active Disease. Geneva: World Health Organization.

World Health Organization [WHO] (2018a). Global Tuberculosis Report 2018. Geneva: World Health Organization.

World Health Organization [WHO] (2018b). Roadmap Towards Ending TB in Children and Adolescents. Geneva: World Health Organization.

Zhou, Y., Du, J., Hou, H.-Y., Lu, Y.-F., Yu, J., Mao, L.-Y., et al. (2017). Application of immunoscore model for the differentiation between active tuberculosis and latent tuberculosis infection as well as monitoring anti-tuberculosis therapy. Front. Cell. Infect. Microbiol. 7, 1-11. doi: 10.3389/fcimb.2017. 00457

Conflict of Interest Statement: The authors declare that the research was conducted in the absence of any commercial or financial relationships that could be construed as a potential conflict of interest.

Copyright (c) 2019 Comella-del-Barrio, Abellana, Villar-Hernández, Jean Coute, Sallés Mingels, Canales Aliaga, Narcisse, Gautier, Ascaso, Latorre, Dominguez and Perez-Porcuna. This is an open-access article distributed under the terms of the Creative Commons Attribution License (CC BY). The use, distribution or reproduction in other forums is permitted, provided the original author(s) and the copyright owner(s) are credited and that the original publication in this journal is cited, in accordance with accepted academic practice. No use, distribution or reproduction is permitted which does not comply with these terms. 